\title{
CRESCIMENTO E DESENVOLVIMENTO DE Physalis angulata Lineu SUBMETIDA AO DÉFICIT HÍDRICO
}

\section{G rowth and development of Physalis angulata Lineu under water deficit}

\author{
Nara Katary dos Reis Souza ${ }^{a}$, Solange Maria Costa de Amorim ${ }^{b}$ \\ a Bióloga, Feira de Santana, BA - Brasil, e-mail: narakatary@ hotmail.com

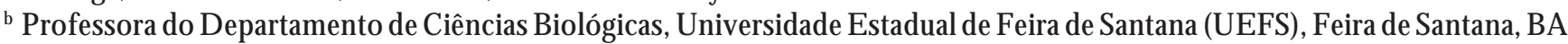 \\ - Brasil, e-mail: samorim@ uefs.br
}

\section{Resumo}

O objetivo deste trabalho foi avaliar o beneficiamento, a colonização micorrízica autócne sobre 0 crescimento e desenvolvimento em Physalis angulata Lineu sob déficit hídrico. Trata-se de uma planta com ciclo de vida anual, chega à sua maturidade aos quatro meses de idade e apresenta ampla utilização na medicina caseira na região semiárida do Nordeste brasileiro. A partir da oitava semana de cultivo, as plantas foram submetidas a dois tratamentos: irrigação diária e déficit hídrico. Physalis mostrou-se pouco eficiente para manter as suas relações ecológicas, como as micorrízicas, na fenofase de frutificação em condições de estresse hídrico.

Palavras-chave: D éficit hídrico. Endomicorriza. Physalis angulata.

\begin{abstract}
The purpose of this work was to evaluate the autocne mycorrhizae improvement under the growth and development in Physalis angulata Lineu under water deficit. This plant has the annual cycle life; its maturity is reached in fourth months of age and presents ample using in the medicine in the region of the semi-arid at the Northeast Brazilian. From the eighth week of culture, the plants had been submitted the two treatments: daily irrigation and water deficit. Physalis revealed little efficient to keep its ecological relations, as the mycorrhizae in fenofase of fructification in conditions of water stress.
\end{abstract}

Keywords: Water deficit. Endomycorrhizal colonization. Physalis angulata. 


\section{INTRODUÇÃO}

A família Solanaceae inclui uma ampla variedade de plantas econômica e farmacologicamente importante como Physalis angulata Lineu, conhecida como camapu. O nome Physalis é oriundo do grego onde "physa" significa bolha ou bexiga, referindo-se ao cálice que encerra seus frutos, comestíveis na maioria das vezes (TO MASSINI et al., 2000).

Pode ser encontrado em diferentes regiões do Brasil. Na Bahia, existem registros nos municípios de Seabra, Água Quente, Mucugê e Rio de Contas. São amplamente utilizados na região semiárida do Nordeste brasileiro no tratamento caseiro do reumatismo crônico, problemas renais, da bexiga e do fígado e também como sedativo, antifebril, antivomitivo e para doenças de pele (MATOS, 2002).

Nas áreas irrigadas do semiárido nordestino, os teores de sais na água de irrigação, intensa evaporação e a deficiência de drenagem provocam problemas de salinidade nos solos, reduzindo 0 crescimento e desenvolvimento das plantas (MACED O, 1988).

A deficiência hídrica em resposta às condições ambientais inerentes à região semiárida é também considerada por Pimentel (1999) e Pimentel et al. (2002) como a maior causadora de redução na produtividade das plantas, alterando o crescimento e a fotossíntese em decorrência da diminuição do teor relativo de água, pressão de turgor e o potencial hídrico celular.

Entre os recursos naturais que podem minimizar as condições severas de falta de água, as micorrizas são associações mutualistas presentes na maioria das plantas vasculares. Por causa da baixa fertilidade natural impostas pelo déficit hídrico no solo, o conhecimento sobre a capacidade das espécies vegetais em formar simbioses com os fungos micorrízicos é de fundamental importância para o sucesso do plantio, podendo determinar a necessidade ou não de inoculação das plantas na fase de formação de mudas (CARNEIRO, 1998).

Os fungos micorrízicos promovem o incremento da absorção de água e elementos minerais, enquanto o fungo obtém compostos de carbono derivados da fotossíntese (ALLEN; MOORE Jr.; CHRISTENSEN, 1980). Dessa forma, a planta tem acesso a uma maior quantidade de água e de elementos nutricionais do solo, incrementa o seu crescimento e desenvolvimento, além de elevar a sua resistência aos estresses bióticos e abióticos (ALARCÓ N; FERRERA; CERRATO, 1999).

Considerando-se que as fases de floração e frutificação, para a maioria das culturas, são as fenofases que apresentam maiores consumos de água, sendo, portanto, as mais sensíveis às condições de déficit hídrico, o objetivo deste trabalho foi avaliar o beneficiamento da colonização micorrízica autóctone sobre o crescimento e desenvolvimento em Physalis angulata Lineu sob déficit hídrico.

\section{MATERIAISE MÉTODOS}

Esta experimentação foi realizada no Laboratório de Ecofisiologia Vegetal da Unidade Experimental do Horto Florestal da Universidade Estadual de Feira de Santana. Sob condições de viveiro, as sementes de Physalis angulata Lineu foram germinadas em bandejas plásticas e as plantas foram cultivadas em sacos plásticos com capacidade de $2 \mathrm{~L}$ do solo franco-argilo-arenoso com as seguintes características químicas: PH H2O (4.3); P (7); K 0.46; Ca (1); Mg (0.5); Al (1.2); Na (0.10); S (2.06); CTC (6.02); V (34.19); M.O . (13.14) segundo o Laboratório de Física e Q uímica do Solo na EMBRAPA - CNPMF- Cruz das Almas, Bahia.

As condições climáticas ambientais médias do experimento foram: precipitação $(28,15 \mathrm{~mm})$, temperaturas máxima $\left(32,85^{\circ} \mathrm{C}\right)$ e mínima $\left(20,35^{\circ} \mathrm{C}\right)$, umidade relativa do ar (40,15 \%), insolação $(10,6 \mathrm{~h})$ e evapotranspiração $(7,35 \mathrm{~mm})$, segundo os registros fornecidos pelo Complexo do Observatório Astronômico Antares - UEFS.

Após 60 dias de cultivo, as plantas foram dispostas em dois blocos experimentais: com hidratação diária (controle) e com submissão ao déficit hídrico pela suspensão da rega. Para impedir a influência da irrigação natural (precipitação), foi instalada uma bancada com barreira artificial com plástico liso transparente número 0,15 sustentado por quatro suportes laterais com $3 \mathrm{~m}$.

As plantas foram cultivadas, inicialmente, sob condições ideais de rega (primeiro mês). Aos dois meses de idade, do primeiro dia de suspensão da rega até o primeiro sintoma de intolerância ao 
déficit hídrico, foram coletados quatro discos em folhas da posição intermediária do caule para 0 monitoramento do conteúdo relativo de água (CRA).

O CRA foi obtido a partir da fórmula CRA = (MF-MS) / (MT-MS) x 100\% segundo Barrs e Weatherley (1962) onde MF, MS e MT representam a massa fresca, massa seca ea massa total, respectivamente (BENICASA, 2003). Durante a fase de cultivo e durante a aplicação dos tratamentos também foram monitorados os números de folhas, flores e de frutos, a altura das plantas e diâmetro do caule.

Para a avaliação da colonização natural endógena dos fungos micorrízicos, em segmentos de dois centímetros de raiz, foi aplicada técnica de Phillips e Hayman (1970) modificada por Grace e Stribley (1991). A avaliação qualitativa da colonização radicular foi realizada segundo Carneiro et al. (1998), em que as amostras analisadas foram categorizadas como alta (todas as estruturas fúngicas), média (hifas e vesículas), baixa (hifas) e sem colonização, mediante a presença de hifas, arbúsculos, vesículas, células reprodutoras na raiz.

O delineamento estatístico foi estruturado em blocos casualizados com oito repetições: irrigação diária (controle) e suspensão de irrigação (déficit hídrico). 0 experimento e os dados obtidos foram analisados através de ANOVA e seguida do teste de Tukey para verificar as diferenças significativas entre as variáveis estudadas, através do programa de estatística Sigmastat, Statistical Software para Windows, versão 2 da Jandel Corporation (1995).

\section{RESULTADOS}

Os primeiros efeitos visíveis do déficit hídrico sobre Physalis angulata Lineu foram observados a partir do quarto dia de tratamento com 0 aparecimento de manchas amarelas nas folhas (principalmente nas mais velhas), seguidas de abscisão foliar.

Conforme a resposta da cultura ao estresse hídrico é possível estabelecer o seu grau de resistência ao déficit de água no solo e definir qual o estádio fenológico mais sensível à disponibilidade de água. 0 déficit hídrico resultou na diminuição do teor relativo de água nas folhas jovens com posição intermediária no caule (folhas maduras e completamente formadas), uma resposta à diminuição do volume celular e desidratação do protoplasto (Figura 1).

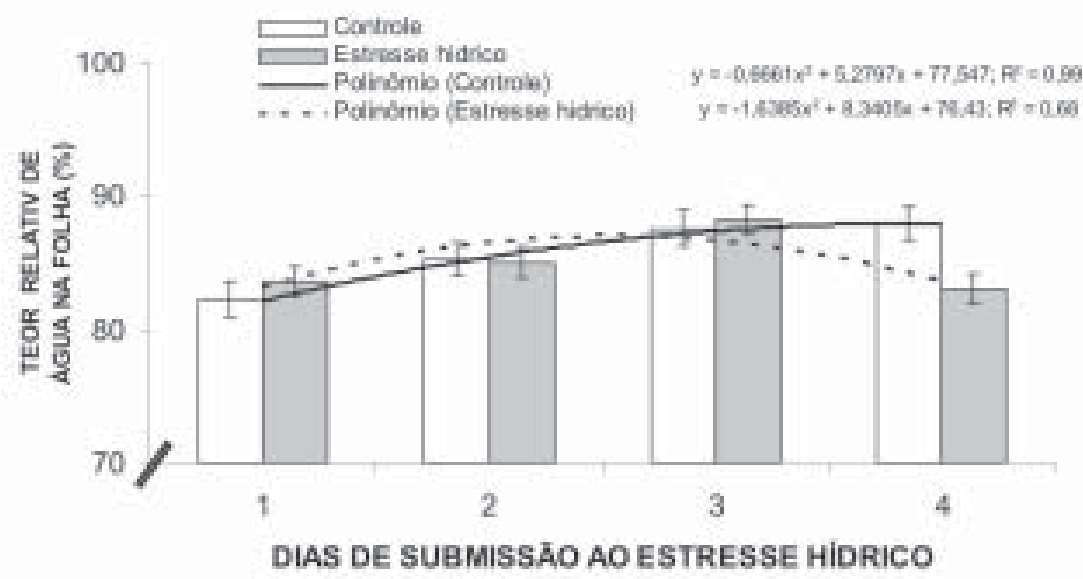

FIGURA 1 - Curvas de regressão polinomial entre teor relativo de água foliar (\%) em Physalis angulata Lineu submetida a quatro dias de déficit hídrico por suspensão da rega. (ANOVA, $\mathrm{P}<$ 0,0001; Tukey, p < 0,05)

A primeira resposta fisiológica ao déficit hídrico é a diminuição da turgescência e, associada a esse evento, a diminuição do crescimento da planta e da produtividade porque as fases de floração e frutificação, para a maioria das culturas, são as que apresentam maiores consumos de água, sendo, portanto, consideradas como fases críticas (CHAVES FILHO; STACCIARINI-SERAPHIN, 2001; LARCHER, 2000; TAIZ; ZEIGER, 2004). 
Magalhães, Mille e Choudhury (1979), mostrando o efeito do déficit hídrico sobre a produção do feijão, confirmam a maior necessidade de água nas fases de floração e frutificação e Q ueiroz, G arcia e Lemos Filho (2002), em experimento com a cultura da berinjela, obtiveram menores valores de altura da planta quando ela foi submetida ao estresse hídrico no início da frutificação.

Com a diminuição do conteúdo de água na folha em Physalis, as células contraíram-se, resultando na redução da área foliar das plantas submetidas ao estresse $\left(12,75 \mathrm{~cm}^{2}\right)$ em relação às plantas irrigadas diariamente $\left(20,475 \mathrm{~cm}^{2}\right)$, uma estratégia para a redução da área de perda de água pela transpiração mediante o suprimento limitado de água pelo solo (Figura 2).

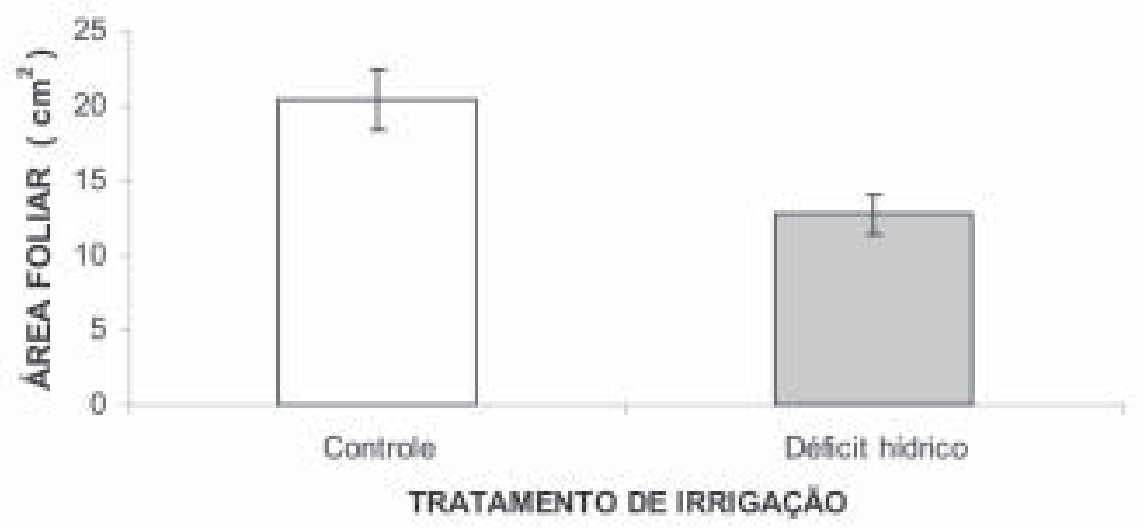

FIGURA 2 - Variação da Área foliar $\left(\mathrm{cm}^{2}\right)$ em Physalis angulata Lineu submetida a quatro dias de déficit hídrico por suspensão da rega (ANOVA, $\mathrm{P}<0,0001$; Tukey, $\mathrm{p}<0,05$ )

Quanto à altura das plantas, não foram evidenciadas respostas diferenciadas entre os tratamentos (ANOVA, $\mathrm{P}<0,001$; Tukey, $\mathrm{p}<0,005$ ), mas foi observada uma acentuada redução do diâmetro do caule nas plantas sob o déficit hídrico em relação às plantas irrigadas diariamente (Figura 3).

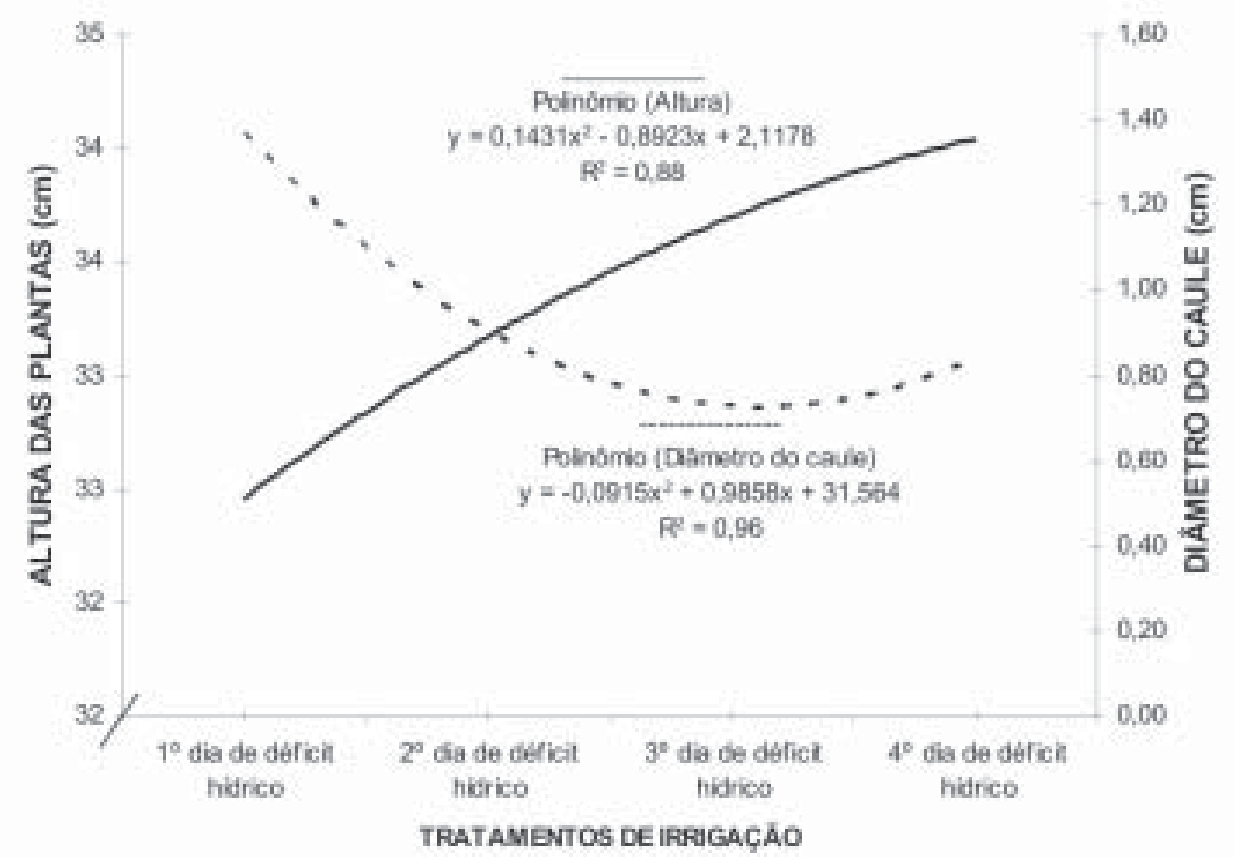

FIGURA 3 - Variação da Altura $(\mathrm{cm})$ e do diâmetro do caule $(\mathrm{cm})$ em Physalis angulata Lineu submetida a quatro dias ao déficit hídrico por suspensão da rega 
O utro efeito detrimental déficit hídrico sobre a taxa de crescimento das plantas foi evidenciado na diminuição do número de folhas no caule (Figura 4), o que certamente, aliado à diminuição da expansão foliar, contribuiu com redução da área fotossintética nas plantas estressadas tal como observaram Távora e Melo (1991) em estudos com a cultura do amendoim submetido a ciclos de deficiência hídrica.

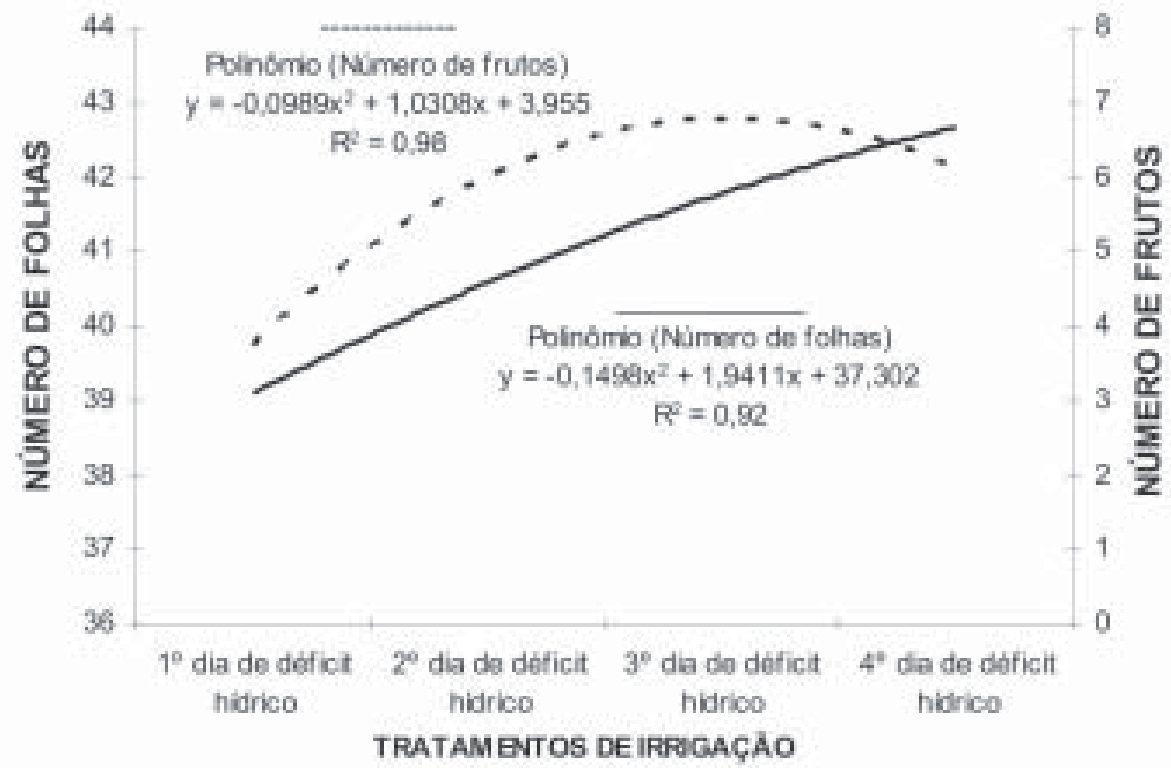

FIGURA 4 - Variação do número de folhas e do número de frutos em Physalis angulata Lineu submetida a quatro dias de déficit hídrico por suspensão da rega

De acordo com Karamanos, Elston e Wadsworth (1982), a ocorrência de estresse hídrico durante a fase vegetativa inicial, provoca redução do crescimento e da superfície fotossintética, ocorrendo, consequentemente, menor número de flores e frutos. Contudo, como o crescimento inicial de Physalis nesse estudo foi sob condições normais de irrigação e os efeitos da supressão das regas pareceram não afetar o início da produtividade no período de déficit hídrico (Figura 5).

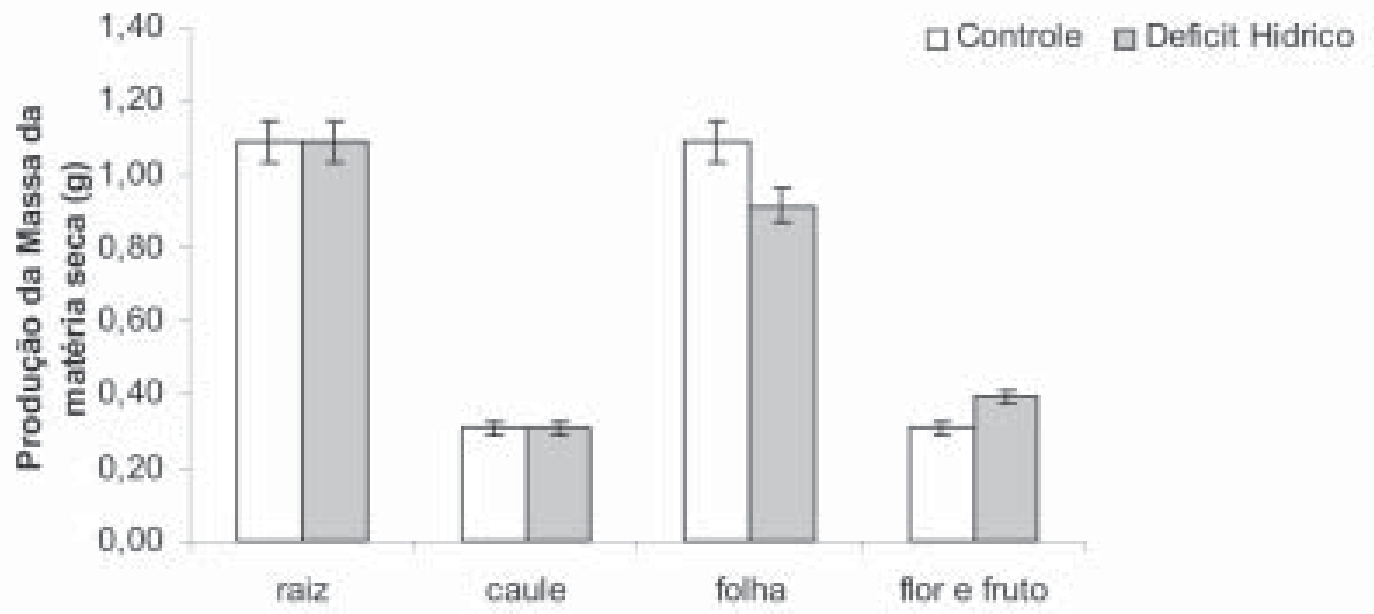

FIGURA 5 - Produção de massa da matéria seca (g) em Physalis angulata Lineu submetida a quatro dias ao déficit hídrico por suspensão da rega. (ANOVA, $\mathrm{P}<0,0001$; Tukey, $\mathrm{p}<0,05)$ 
Segundo Queiroz, Garcia e Lemos Filho (2002), o início da frutificação é um período sensível ao déficit hídrico em cultura de berinjela. Os efeitos negativos do estresse hídrico sobre a frutificação neste estudo foram observados no quarto dia de tratamento, sugerindo que Physalis angulata foi eficiente na utilização da água estocada nos tecidos durante os três primeiros dias de estresse hídrico (Figura 4) e que a partir do quarto dia de déficit o solo já entrava em estado de deficiência hídrica.

Para superar o estresse por déficit de água, Physalis teve a produção de massa da matéria seca radicular aumentada (Figura 5) devido, provavelmente, à estocagem de assimilados na parte subterrânea em resposta da redução da expansão foliar e, consequentemente, diminuição de consumo de carbono e energia. As raízes, nessas circunstâncias, tendem a crescer até as camadas mais úmidas do solo até que o suprimento de água seja exaurido no ambiente (TAIZ; ZEIGER, 2004).

Entretanto, durante o déficit hídrico os assimilados são dirigidos para os frutos e distanciados das raízes, o que explica a maior sensibilidade das plantas ao estresse hídrico durante a fase reprodutiva (TAIZ; ZEIGER, 2004).

Segundo Babalola (1980) e Leite, Rodrigues e Virgens Filho (1999, 2000), a translocação de fotoassimilados para as raízes é comprometida em condições de déficit e a exportação de material assimilado da folha para outros órgãos da planta pode ser comprometida, ocasionando decréscimos do crescimento e produção do vegetal.

Neste estudo, o direcionamento dos assimilados para frutos durante o déficit hídrico pareceu afetar mais efetivamente a colonização micorrízica e, dessa forma, a planta teve acesso a uma menor quantidade de água e de elementos nutricionais do solo e o seu crescimento diminuiu.

A categorização da colonização micorrízica nas raízes das plantas estressadas foi baixa com relação às plantas irrigadas diariamente. Nos 200 segmentos radiculares das plantas irrigadas diariamente, mais de $80 \%$ das lâminas avaliadas apresentaram todos os componentes da colonização micorrízica como: esporos intracelulares; células reprodutoras e hifas intra e extrarradiculares, enquanto os segmentos radiculares das plantas submetidas ao déficit hídrico, 70\% apresentaram apenas hifas intra e extrarradiculares. Provavelmente, o baixo grau de colonização associou-se à competição por assimilados entre a parte aérea da planta (frutos) e as raízes (Figura 6) e o fungo não conseguiu obter compostos de carbono derivados da fotossíntese suficiente para manter a simbiose em grau elevado.

0 diferencial no grau de interação entre o fungo e Physalis nos tratamentos aplicados neste estudo pode, também, estar relacionado ao genótipo da planta (JANOS, 1988), suprimento de P para as suas raízes e, sobretudo, ao grau de dependência da planta ao fungo durante 0 déficit hídrico (ALARCO N; FERRERA-CERRATO, 1999; AMORIM; PAIM; SILVA, 2004; KOIDE, 1991).

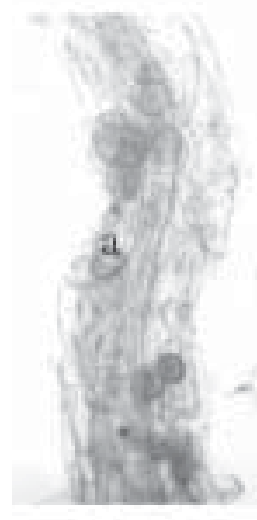

1

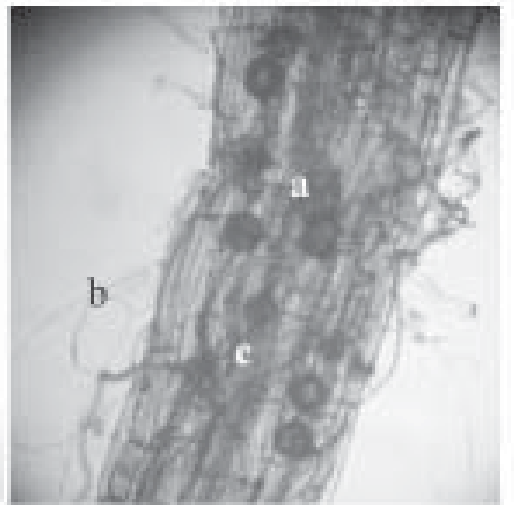

2

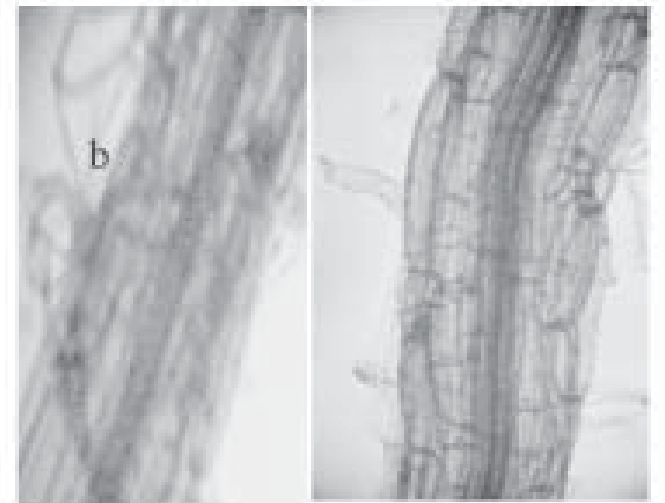

3
4

FIG URA 6 - Morfologia da colonização micorrízica em Physalis angulata Lineu submetida à irrigação diária (1 e 2; a=esporos, b=hifas externas, $\mathrm{c}=$ hifas internas) e quatro dias ao déficit hídrico por suspensão da rega (3 e 4; b=hifas externas). Escala 10um e zoom 3.2. Imagem Digital Polaroid 


\section{CONCLUSÕES}

Physalis angulata Lineu apresenta estratégias relevantes para utilizar a água disponível no solo imposta pela situação de déficit hídrico.

O limite crítico (dias de estresse) para P hysalis angulata Lineu superar o déficit hídrico na fenofase de frutificação é aproximadamente quatro dias e esta planta mostra-se pouco eficiente para manter as suas relações ecológicas, como as micorrízicas, na fenofase de frutificação em condições de estresse hídrico.

\section{REFERÊNCIAS}

ALARCÓN, A.; FERRERA-CERRATO, R. Manejo de la micorriza arbuscular en sistemas de propagación de plantas frutícolas arbuscular mycorrhizae. Management on Fruit Plant Propagation Systems. Terra, Rio de Janeiro, v. 17, n. 3, p. 179-191, 1999.

ALLEN, M. F. T. S.; MO O RE Jr., Y. M. C.; CHRISTENSEN, M. Phytohormone changes in Bouteloua gracilis infected by vesiculararbuscular mycorrhizae. I. Cytokinin increases in the host plant. Can. J. Botany, Canada, v. 58, n. 3, p. 371-374, 1980.

AMO RIM, S. M. C. de; PAIM, A. C. B.; SILVA, M. G. Estudo ecofisiológico sobre endomicorrizas: 0 efeito do déficit hídrico sobre a colonização endomicorrízica em duas espécies vegetais típicas da região semi-árida do Nordeste. Revista Biotecnologia Ciência e Desenvolvimento, Brasília, n. 33, p. 23-26, 2004.

BABALOLA, O. Water relations of three cowpea cultivars [V igna unguiculata (L.) Walp]. Plant and Soil, v. 56, n. 1, p. 59-69, 1980.

BARRS, H. D.; WEATHERLEY, P. E. A re-examination of the relative turgidity technique for estimating water deficits in leaves. Aust. J. Biol. Sci., Melbourne, v. 15, p. 413-428, 1962.

BENICASA, M. M. P. Análise de crescimento de plantas (noções básicas). 2. ed. Jaboticabal: FUNEP, 2003.

CARNEIRO, M. A. C. et al. Micorriza arbuscular em espécies arbóreas e arbustivas nativas de ocorrência no Sudeste do Brasil. Cerne, Lavras, v. 4, n. 1, p. 129-145, 1998.

CHAVES FILHO, J. T.; STACCIARINI-SERAPHIN, E. Alteração no potencial osmótico e teor de carboidratos solúveis em plantas jovens de lobeira (Solanum lycocarpum St.-Hil.) em resposta ao estresse hídrico. Rev. Bras. Bot., São Paulo, v. 24, n. 2, p. 199-204, 2001.

GRACE, C.; STRIBLEY, D. P. A. Safer procedure for routine staining of vesicular arbuscular mycorrhizal fungi. Mycology, Stockholm, v. 95, n. 9, p. 1160-1162, 1991.

JANOS, D. P. Mycorrhizal applications in tropical forestry: are temperate-zone approaches? In: NG, S. P. (Ed.). Trees and mycorrhizas. Kaula Lumpur: Forest Research Institute of Malaysia, 1988. p. 133-188.

KARAMANOS, A. J.; ELSTON, J.; WAD SWORTH, R. M. Water stress and leaf growth of field beans (V icia faba, L.) in the field: water potentials and laminar expansion. Ann. Botany, New York, v. 49, n. 6, p. 815-826, 1982.

KO IDE, R. T. Nutrient supply, nutrient demand and plant response to mycorrhizal infection. N ew Phytologist, Cambridge, v. 117, n. 3, p. 365-386, 1991.

LARCHER, W. Ecofisiologia vegetal. São Carlos: RIMA, 2000. 
LEITE, M. L.; RODRIG UES, J. D.; VIRGENS FILHO, J. S. Efeitos do déficit hídrico sobre a cultura do caupi [V igna unguiculata (L.) Walp], cv. EMAPA-821. II - Análise de Crescimento. Rev. Agric., Piracicaba, v. 74, n. 3, p. 351-370, 1999.

. Efeitos do déficit hídrico sobre a cultura do caupi, cv. EMAPA-821. III - Produção. Rev. Ägric., Piracicaba, v. 75, n. 1, p. 9-20, 2000.

MACÊD O, L. de S. Salinidade em áreas irrigadas. Comunicado Técnico, n. 38, p. 1-11, 1988.

MAGALHÃES, A. A.; MILLE, A. A.; CHOUD HURY, E. N. Efeito do déficit fenológico de água sobre a produção de feijão. IICA , Turrialba, Costa Rica, v. 29, n. 4, p. 269-73, 1979.

MATOS, F. J. A. Plantas medicinais: guia de seleção e emprego de plantas usadas em fitoterapia no Nordeste do Brasil. 2. ed. Fortaleza: Ed. UFC, 2002.

PHILLIPS, J. M.; HAYMAN, D. S. Improved procedures for clearing roots and staining parasitic, and vesicular-arbuscular mycorrhizal fungi for rapid assessment of infection. British Mycological Society Transactions, Cambridge, v. 55, n. 1, p. 158-160, 1970.

PIMENTEL, C. Relações hídricas em dois híbridos de milho sob dois ciclos de deficiência hídrica. Pesq. Agropecu. Brás, Rio de Janeiro, v. 34, n. 11, p. 2021-2027, 1999.

PIMENTEL, C. et al. Tolerância protoplasmática foliar à seca, em dois genótipos de caupi cultivadas em campo. Rev. Univ. Rural, Ser. Cienc. Vida, Rio de Janeiro, v. 22, n. 1, p. 07-14, 2002.

QUEIROZ, C. G. S.; GARCIA Q. S.; LEMO S FILHO, J. P. A tividade fotossintética e peroxidação de lipídeos de membrana em plantas de arroeira-do-sertão sob estresse hídrico e após reidratação. Rev. Bras. Fisiol. Veg., Londrina, v. 14, n. 1, p. 59-63, 2002.

TAIZ, L.; ZEIGER, E. Fisiologia vegetal. 3. ed. Porto Alegre: Artmed, 2004.

TÁVORA, F. J. A. F.; MELO, F. I. O. Respostas de cultivares de amendoim a ciclos de deficiência hídrica: crescimento vegetativo, reprodutivo e relações hídricas. Ciênc. Agron., Fortaleza, v. 22, n. 1, p. 47-60, 1991.

TÁVORA, F. J. A. F.; FERREIRA, R. G.; HERNAND EZ, F. F. F. Crescimento e relações hídricas em plantas de goiabeira submetidas a estresse salino com NaCl. Rev. Bras. Frutic., Jaboticabal, v. 23, n. 2, p. 441-446, 2001.

TOMASSINI, T. C. B. et al. G ênero Physalis: uma revisão sobre vitaesteróides. Química Nova, São Paulo, v. 23, n. 1, p. 47-57, 2000.

Recebido: $27 / 09 / 2008$ Received: 09/27/ 2008

Aprovado: $12 / 02 / 2008$ A pproved: 02/ 12/ 2008

Revisado: $18 / 08 / 2009$ Reviewed: 08/ 18/2009 